\title{
The application of transbronchial cryobiopsy in interstitial lung disease: a prospective, multicenter, real-world study
}

\author{
Xiaobo Chen ${ }^{1 \#}$, Jing $\mathrm{Li}^{2 \#}$, Fengming Luo ${ }^{3 \#}$, Feng Tao ${ }^{4}$, Xiaoju Zhang ${ }^{5}$, Youru $\mathrm{Wu}^{6}$, Ping $\mathrm{Xu}^{7}$, \\ Mingyao Ke ${ }^{8}$, Fa Long ${ }^{9}$, Laiyu Liu ${ }^{10}$, Liping Lv ${ }^{11}$, Huai Liao ${ }^{12}$, Ye Gu ${ }^{13}$, Zhiguang Liu ${ }^{14}$, Xiaoming Tan ${ }^{15}$, \\ Shuliang Guo ${ }^{16}$, Yi Hu ${ }^{17}$, Huaping Yang ${ }^{18}$, Yong Zhou ${ }^{19}$, Hongmei Zhou ${ }^{20}$, Yongshun Ye ${ }^{21}$, Difei Chen ${ }^{1}$, \\ Shiyue $\mathbf{L i}^{1}$
}

${ }^{1}$ State Key Laboratory of Respiratory Disease, National Clinical Research Center for Respiratory Disease, Guangzhou Institute of Respiratory Health, The First Affiliated Hospital of Guangzhou Medical University, Guangzhou, China; ${ }^{2}$ Department of Respiratory and Critical Care Medicine, Guangdong Provincial People's Hospital/Guangdong Academy of Medical Sciences, Guangzhou, China; ${ }^{3}$ Department of Respiratory and Critical Care Medicine, West China Hospital, Sichuan University, Chengdu, China; ${ }^{4}$ Department of Respiratory and Critical Care Medicine, The First Hospital of Jiaxing/First Affiliated Hospital of Jiaxing University, Jiaxing, China; ${ }^{5}$ Department of Respiratory and Critical Care Medicine, Henan Provincial People's Hospital, Zhengzhou University People's Hospital, Zhengzhou, China; ${ }^{6}$ Department of Respiratory and Critical Care Medicine, Mianyang Central Hospital, Mianyang, China; ${ }^{7}$ Department of Respiratory and Critical Care Medicine, Peking University Shenzhen Hospital, Shenzhen, China; ${ }^{8}$ Department of Respiratory Centre, The Second Affiliated Hospital of Xiamen Medical College, Xiamen, China; ${ }^{9}$ Department of Pulmonary and Critical Care Medicine, University of Chinese Academy of Sciences Shenzhen Hospital, Shenzhen, China; ${ }^{10}$ Chronic Airways Diseases Laboratory, Department of Respiratory and Critical Care Medicine, Nanfang Hospital, Southern Medical University, Guangzhou, China; ${ }^{11}$ Endoscopy Center, Anhui Chest Hospital, Hefei, China; ${ }^{12}$ Department of Respiratory and Critical Care Medicine, First Affiliated Hospital of Sun Yat-sen University, Guangzhou, China; ${ }^{13}$ Endoscopy Center, Shanghai Pulmonary Hospital, Tongji University School of Medicine, Shanghai, China; ${ }^{14}$ Department of Respiratory and Critical Care Medicine, Hunan Provincial People's Hospital, The First Affiliated Hospital of Hunan Normal University, Changsha, China; ${ }^{15}$ Department of Respiratory Medicine, Renji Hospital, School of Medicine, Shanghai Jiaotong University, Shanghai, China; ${ }^{16}$ Department of Respiratory and Critical Care Medicine, The First Affiliated Hospital of Chongqing Medical University, Chongqing, China; ${ }^{17}$ Department of Pulmonary and Critical Care Medicine, The Central Hospital of Wuhan, Tongji Medical College, Huazhong University of Science and Technology, Wuhan, China; ${ }^{18}$ Department of Pulmonary and Critical Care Medicine, Xiangya Hospital Central South University, Changsha, China; ${ }^{19}$ Center for Respiratory Endoscopy Diagnosis and Treatment, Xi'an Chest Hospital, Xi'an, China; ${ }^{20}$ Department of Pulmonary and Critical Care Medicine, Tianyou Hospital affiliated with Tongji University, Shanghai, China; ${ }^{21}$ Department of Pulmonary and Critical Care Medicine, Huizhou Municipal Central Hospital, Huizhou, China

Contributions: (I) Conception and design: S Li, X Chen; (II) Administrative support: S Li, X Chen; (III) Provision of study materials or patients: All authors; (IV) Collection and assembly of data: All authors; (V) Data analysis and interpretation: X Chen, Y Ye, D Chen; (VI) Manuscript writing: All authors; (VII) Final approval of manuscript: All authors.

\#These authors contributed equally to this work.

Correspondence to: Shiyue Li. State Key Laboratory of Respiratory Disease, National Clinical Research Center for Respiratory Disease, Guangzhou Institute of Respiratory Health, The First Affiliated Hospital of Guangzhou Medical University, 151 Yanjiang Rd., Guangzhou 510120, China. Email: lishiyue@188.com.

Background: Transbronchial cryobiopsy (TBCB) has been widely used to diagnose interstitial lung disease (ILD). Existing reports on TBCB in ILD are mostly single-center prospective or retrospective studies but rarely multicenter prospective real-world studies. We explored the diagnostic efficiency and safety of TBCB in ILD in a real world setting.

Methods: A prospective, multicenter, real-world study was conducted to analyze the data of patients with unclarified ILD who underwent TBCB in 20 hospitals in China from October 2018 to October 2019. The results of the pathological and multidisciplinary discussion (MDD) diagnosis and complications related to TBCB were then analyzed.

Results: A total of 373 patients were enrolled in this study, including 194 males and 179 females, with an average age of 52.6 \pm 12.4 years. None of the patients had severe hemorrhaging, and the incidence of 
pneumothorax was $4.8 \%$. The proportions of definitive, possible, and unclassified pathological diagnoses were $62.5 \%$, 5.6\%, and 31.9\%, respectively. The overall diagnostic yield of MDD was $63.5 \%$. There were 237 patients with a definitive diagnosis of MDD and 136 patients with an unclarified MDD diagnosis. The cooling gas pressure, freezing durations, number of specimens, maximum lengths of specimens, and specimen sizes varied significantly between the definitive and unclarified MDD diagnoses.

Conclusions: In China, the application of TBCB in ILD is generally safe, and its diagnostic efficiency is acceptable. Using a 1.9-mm cryoprobe to collect five samples would achieve a better positive diagnostic rate for TBCB in ILD, without a significant increase in complication risk.

Trial Registration: ClinicalTrials.gov; date of registration: 09/25/2018; registration number: NCT03704233; URL: clinicaltrials.gov.

Keywords: Transbronchial cryobiopsy (TBCB); interstitial lung disease (ILD); efficiency; safety; real-world

Submitted Jul 02, 2021. Accepted for publication Sep 22, 2021.

doi: $10.21037 / \mathrm{atm}-21-3461$

View this article at: https://dx.doi.org/10.21037/atm-21-3461

\section{Introduction}

Interstitial lung diseases (ILDs) are a heterogeneous group of diffuse parenchymal lung diseases characterized by varying histopathologic patterns of inflammation and fibrosis. Transbronchial cryobiopsy (TBCB) is required to supply adequate tissue samples for a histological diagnosis and multidisciplinary diagnosis (MDD) of ILD. Because of the complex composition of the etiology and heterogeneous pathological alterations in different lung regions of ILD, it is a challenge to define the clinical value of TBCB with surgical lung biopsy (SLB) in a randomized controlled study. Previous studies have shown that the application value of TBCB in the diagnosis of ILD is controversial (1-4). Additional studies are needed to assess the impact of the technical aspects of TВСВ during ILD diagnostic yields and safety (5).

A real-world study could better reflect the actual clinical application value of TBCB in ILD. In particular, existing TBCB reports are mostly prospective or retrospective, single-center studies. However, no real-world, multicenter studies in China have been conducted to clarify the efficiency and safety of TBCB in ILD. We thus aimed to explore the efficiency and safety of TBCB in ILD in the real-world setting. We present the following article per the STROBE reporting checklist (available at https://dx.doi. org/10.21037/atm-21-3461).

\section{Methods}

\section{Patient selection}

This study used large-scale, prospective, multicenter, real- world observational methods to collect data from patients with suspected ILD who needed a TBCB procedure from 20 hospitals across 12 provinces or municipalities in China from October 15, 2018, to October 15, 2019. The enrolled patients were required to meet the following inclusion criteria: (I) diagnosed with ILD based on complete clinical data (including medical history, serology, pulmonary function tests, and high-resolution computed tomography); and (II) required TBCB to confirm the diagnosis after a multidisciplinary discussion (MDD). The exclusion criteria were the following: (I) abnormal blood coagulation function or thrombocytopenia $\left(<50 \times 10^{9} / \mathrm{L}\right)$ and (II) refusal to participate in this study. The patients were continuously enrolled in their group and their data were recorded, including clinical data, anesthesia methods, freezing duration, specimen size, complications, pathological diagnosis, and MDD diagnostic results. The Ethics Committee of the First Affiliated Hospital of Guangzhou Medical University, Guangdong Province, China, approved this study (No. 201830). Informed consent was obtained from each patient after a full explanation of the purpose of this study. All procedures performed in this study involving human participants were in accordance with the Declaration of Helsinki (as revised in 2013).

\section{Surgical procedures}

Patients underwent bronchoscopy with a flexible bronchoscope (Olympus BF-260 or IT260 bronchoscope, Olympus, Japan) under anesthesia (methods of anesthesia 
varied between patients). First, in vitro tests with a cryosurgical unit $\left(\right.$ ERBOKRYO $^{\circledR}$ CA or ERBECRYO ${ }^{\circledR} 2$; Erbe Elektromedizin GmbH., Tübingen, Germany) and cryoprobe (1.9- or 2.4-mm diameter; Erbe) were performed to confirm normal working conditions. The cooling gas source was carbon dioxide, and the normal cooling gas pressure ranged from 40-60 bar in the cryosurgical system (ERBOKRYO ${ }^{\circledast} \mathrm{CA}$; Erbe) and from $45-65$ bar in the cryosurgical system (ERBECRYO 2; Erbe). A balloon catheter (Arndt Endobronchial Blocker Set, C-AEBS9.0-78-SPH-AS, Cook Medical, Bloomington, USA) was placed preventively in the bronchus of the biopsy site. The cryoprobe was then advanced to the biopsy site through the working channel of the bronchoscope. The cryoprobe was placed approximately 1-2 $\mathrm{cm}$ away from the pleura under fluoroscopy or endobronchial ultrasound guidance. In the cases with no navigation guidance, the cryoprobe was then sent to the biopsy site according to the high-resolution computed tomography images. Once resistance was encountered, the cryoprobe was pulled back $1-2 \mathrm{~cm}$. The freezing time was adjusted according to the diameter of the cryoprobe and the size of specimen. After cryocooling, the bronchoscope and cryoprobe were pulled back simultaneously. For the measurement of bleeding, negative pressure suction was performed with a bronchoscope. If bleeding persisted, the balloon catheter was inflated to occlude the bronchus of the biopsy site. We independently collected and measured the specimens. The size of the specimen and any complications of bleeding and pneumothorax were recorded. The TBCB operations were terminated when severe bleeding or pneumothorax occurred during the procedures.

\section{Classification of bleeding severity}

The degree of the severity of bleeding in patients was classified as described in previous literature (6): grade $0=$ no bleeding; grade $1=$ bleeding stopped with negative pressure suction without other hemostatic measures; grade $2=$ bleeding which was stopped by with local injection of frozen saline, epinephrine, the balloon occlusion, or intravenous hemostatic drugs; and grade $3=$ severe bleeding that could not be controlled by endoscopy, causing instability of hemodynamics and respiratory function, requiring either vascular intervention or surgical intervention or admission to the intensive care unit (ICU). Grades 0-1 were considered to be mild bleeding, grade 2 was moderate bleeding, and grade 3 was severe bleeding.

\section{Diagnosis}

The specimens were obtained and placed in $10 \%$ formalin solution for fixation and subsequent pathological examination. Two pathologists with extensive experienced in the pathological diagnosis of ILD then reviewed the samples. The pathological diagnosis was classified into three 3 categories based on diagnostic intensity: (I) definitive pathological diagnosis, (II) possible pathological diagnosis and (III) unclassified diagnosis. The final ILD diagnosis was obtained through an MDD with clinicians, radiologists, and pathologists.

\section{Statistical analysis}

The SPSS 16.0 statistical software (IBM SPSS Inc., Chicago, IL, USA) was used for the statistical analysis. The normally distributed measurement data were are presented as means \pm standard deviations, and while the non-normally distributed measurement data were are presented as median values. Comparison of the incidences of bleeding and the diagnosis diagnostic yield of MDD between groups was performed by using the Chi-square $\left(\chi^{2}\right)$ test. The MannWhitney U rank-sum test was used to compare the cooling gas pressure, the number of samples, and the size of the sample between the definitive diagnosis of MDD and the unclassified diagnosis groups. The $\chi^{2}$ test was used to compare of the navigation ratio used between the 2 groups. Comparison of the incidence of bleeding, pathology diagnosis yield, and MDD diagnostic yield between the 2 groups was performed with cryoprobes of $1.9-\mathrm{mm}$ and 2.4-mm using the $\chi^{2}$ test. Comparison of the incidence of pneumothorax between the 2 groups was performed using Fisher's exact test. A P value $<0.05$ was considered statistically significant.

\section{Results}

\section{Demographic characteristics of the patients}

This study enrolled 373 patients from 20 hospitals in 12 provinces or municipalities, including 194 males and 179 females, with an average age of $52.6 \pm 12.4$ years (range, 14-81 years). Among them, 118 patients (31.6\%) had underlying diseases (e.g., hypertension, diabetes, heart disease, and cerebrovascular accident). In the arterial blood gas test, the $\mathrm{pH}$ of the patients, the partial pressure of oxygen in arterial blood $\left(\mathrm{PaO}_{2}\right)$, and the partial pressure of carbon dioxide in arterial blood $\left(\mathrm{PaCO}_{2}\right)$ were 
Table 1 Technical parameters applied in transbronchial cryobiopsy

\begin{tabular}{lcc}
\hline Technical parameters & Median & Range \\
\hline Cooling gas pressure (bar) & 58.0 & $40-65$ \\
Freezing duration (seconds) & 5.0 & $2-12$ \\
Number of specimens & 4.0 & $1-5$ \\
Method of anesthesia, $\mathrm{n}(\%)$ & & \\
General intravenous anesthesia & $328(87.9)$ & - \\
Local anesthesia & $45(12.1)$ & - \\
Ventilation method, $\mathrm{n}(\%)$ & & \\
Rigid bronchoscope & $89(23.9)$ & - \\
Tracheal intubation & $203(54.4)$ & - \\
Laryngeal mask & $36(9.7)$ & - \\
Navigation method, $\mathrm{n}(\%)$ & & - \\
X-ray guidance & $184(49.3)$ & - \\
Ultrasound guidance & $62(16.6)$ & - \\
Total & $246(66)$ & - \\
\hline
\end{tabular}

$7.41 \pm 0.03,86.3 \pm 23.7$, and $39.9 \pm 5.4 \mathrm{mmHg}$, respectively. In the pulmonary function tests, the predicted values of forced expiratory volume in the first second (FEV1), forced vital capacity (FVC), and the diffusing capacity of the lungs for carbon monoxide (DLCO) of the patients were $70.6 \% \pm 25.6 \%, 71.1 \% \pm 25.1 \%$, and $53.5 \% \pm 28.8 \%$, respectively.

\section{Result of TBCB}

There were 328 (87.9\%) patients who underwent ТВСВ under general intravenous anesthesia, including 89 (23.9\%) patients who received rigid bronchoscopy, 203 (54.4\%) patients who underwent tracheal intubation, and $36(9.7 \%)$ patients who received a laryngeal mask for ventilation. The remaining $45(12.1 \%)$ patients were conscious and under sedation and analgesia during the TBCB procedures.

There were $246(66.0 \%)$ patients whose procedures used navigation tools, including $\mathrm{C}$-arm X-ray machines and endobronchial ultrasound probes, to guide the ТВСВ procedures. All patients had balloon occlusion catheters to prevent bleeding.

The normal pressure of the cooling gas was between 40 and 65 bar, with a median of 58 bar. The freezing time was between 2 and 12 seconds, with a median of 5 seconds. The number of specimens obtained from each patient range from 1 to 5 , with a median of 4 . From the 373 patients in this study, 1,383 specimens were obtained, with the specimen size ranging from 0.5 to $176 \mathrm{~mm}^{2}$ and a median size of $16.8 \mathrm{~mm}^{2}$ (Table 1).

In all, 158 (42.4\%) patients had mild bleeding, and 215 $(57.6 \%)$ had moderate bleeding. No life-threatening severe bleeding was found among the patients. Among the 19 (5.1\%) patients who developed pneumothorax, 17 patients required closed chest drainage, while the other 2 were treated conservatively. All pneumothorax patients recovered within a week. In this study, the exacerbation of ILD was not recorded. There were $233(62.5 \%)$ patients with a definitive pathological diagnosis, $21(5.6 \%)$ patients with a possible pathological diagnosis, and 119 (31.9\%) patients with an unclarified pathological diagnosis. Eventually, 237 $(63.5 \%)$ patients obtained a definitive diagnosis through MDD (Table 2).

The spectrum of definitive pathological diagnosis and the final MDD diagnoses for each biopsy type are shown in Table 3 and Table 4 respectively. However, among the 233 patients $(62.5 \%)$ with a definitive pathological diagnosis, there were still 21 patients (5.6\%) who only received a possible pathological diagnosis and 119 patients (31.9\%) had an unclassified pathological diagnosis (Table 3). Among the $237(63.5 \%)$ patients who had a definitive final diagnosis through MDD, there were 136 patients (36.5\%) with an unclarified MDD diagnosis (Table 4).

\section{Comparison of technical parameters of TBCB in cases with a definitive diagnosis of MDD and an unclarified diagnosis of $M D D$}

After dividing the cases into 2 groups according to their definitive MDD diagnosis $(\mathrm{n}=237)$ and unclarified MDD diagnosis $(n=136)$, the cooling gas pressure, freezing duration, specimen size, and navigation tools used between the 2 groups were compared. The results showed that the cooling gas pressure, the freezing duration, number of specimens obtained, the maximum diameter of specimen, and the specimen sizes of the two groups varied significantly $(\mathrm{P}<0.001$, Table 5).

\section{Comparison of complications and diagnostic efficiencies of 1.9-mm cryoprobe and 2.4-mm cryoprobe}

In this study, 326 patients $(87.4 \%)$ underwent ТВСВ with a $1.9-\mathrm{mm}$ cryoprobe (1.9-mm cryoprobe group) and 47 patients $(12.6 \%)$ underwent TBCB with a $2.4-\mathrm{mm}$ 
Table 2 Clinical characteristics of the patient, complications, and diagnostic efficiency of transbronchial cryobiopsy

\begin{tabular}{lc}
\hline Clinical characteristics of the & Mean \pm standard deviation \\
patients & $179 / 194$ \\
\hline Sex (female/male) & $52.6 \pm 12.4$ \\
Age (years) & $7.41 \pm 0.03$ \\
$\mathrm{pH}$ & $39.9 \pm 5.4$ \\
$\mathrm{PaCO}_{2}$ (mmHg) & $86.3 \pm 23.7$ \\
$\mathrm{PaO}_{2}$ (mmHg) & $70.6 \pm 25.6$ \\
$\mathrm{FEV} 1$ (pre\%) & $71.1 \pm 25.1$ \\
$\mathrm{FVC}$ (pre\%) & $53.5 \pm 28.8$ \\
$\mathrm{DLCO}$ (pre\%) & \\
Bleeding severity, $\mathrm{n}(\%)$ & $71(19.0)$ \\
Grade 0 & $87(23.3)$ \\
Grade 1 & $215(57.6)$ \\
Grade 2 & 0 \\
Grade 3 & $119(31.9)$ \\
Pneumothorax, $\mathrm{n}$ (\%) & $237(63.5)$ \\
Pathological diagnosis, $\mathrm{n}(\%)$ & $233(62.5)$ \\
Clear pathological diagnosis & $21(5.6)$ \\
Possible pathological diagnosis & \\
Unknown pathological diagnosis & $\mathrm{n}$ \\
\hline
\end{tabular}

$\mathrm{pH}$, potential of hydrogen; $\mathrm{PaCO}_{2}$, partial pressure of carbon dioxide in arterial blood; $\mathrm{PaO}_{2}$, pressure of oxygen in arterial blood; FEV1, forced expiratory volume in the first second; FVC, the forced vital capacity; DLCO, the diffusing capacity of the lungs for carbon monoxide; MDD, multidisciplinary discussion.

Table 3 Histopathological diagnosis of patients undergoing TBCB

\begin{tabular}{lc}
\hline Pathological diagnosis & Case $(\%)$ \\
\hline Definitive pathological diagnosis & $77(20.6)$ \\
UIP & $48(12.9)$ \\
NSIP & $13(3.5)$ \\
OP & $14(3.8)$ \\
PAP & $10(2.7)$ \\
LAM & $18(4.8)$ \\
Lung cancer & $9(2.4)$ \\
Pneumonia &
\end{tabular}

Table 3 (continued)
Table 3 (continued)

\begin{tabular}{|c|c|}
\hline Pathological diagnosis & Case $(\%)$ \\
\hline Bullous emphysema & $7(1.9)$ \\
\hline Sarcoidosis & $6(1.6)$ \\
\hline Pneumoconiosis & $5(1.3)$ \\
\hline TB & $4(1.1)$ \\
\hline DIP & $3(0.8)$ \\
\hline HP & $3(0.8)$ \\
\hline RB-ILD & $2(0.5)$ \\
\hline ACFE & $2(0.5)$ \\
\hline PCP & $1(0.3)$ \\
\hline PIE & $1(0.3)$ \\
\hline $\mathrm{LCH}$ & $1(0.3)$ \\
\hline PAM & $1(0.3)$ \\
\hline Lipid pneumonia & $1(0.3)$ \\
\hline PO & $1(0.3)$ \\
\hline Cryptococcosis & $1(0.3)$ \\
\hline Giant cell interstitial pneumonia & $1(0.3)$ \\
\hline $\mathrm{IPH}$ & $1(0.3)$ \\
\hline Aspiration pneumonia & $1(0.3)$ \\
\hline Congenital cystic adenomatoid malformations & $1(0.3)$ \\
\hline Pulmonary intravascular large B-cell lymphoma & $1(0.3)$ \\
\hline \multicolumn{2}{|l|}{ Possible pathological diagnosis } \\
\hline UIP & $5(1.3)$ \\
\hline HP & $2(0.5)$ \\
\hline NSIP & $4(1.2)$ \\
\hline DIP & $1(0.3)$ \\
\hline OP & $1(0.3)$ \\
\hline PAP & $2(0.5)$ \\
\hline RB-ILD & $1(0.3)$ \\
\hline TB & $2(0.5)$ \\
\hline PCP & $1(0.3)$ \\
\hline Sarcoidosis & $2(0.5)$ \\
\hline Unknown diagnosis & $119(31.6)$ \\
\hline \multicolumn{2}{|c|}{$\begin{array}{l}\text { UIP, usual interstitial pneumonia; NSIP, non-specific interstitia } \\
\text { pneumonia; DIP, desquamative interstitial pneumonia } \\
\text { HP, hypersensitivity pneumonitis; PLAM, pulmonary } \\
\text { lymphangiomyomatosis; OP, organizing pneumonia; PAP } \\
\text { pulmonary alveolar proteinosis; PCP, pneumocystis pneumonia } \\
\text { PIE, pulmonary infiltration with eosinophilia; RB-ILD, respiratory } \\
\text { bronchiolitis-interstitial lung disease; LCH, langerhans cel } \\
\text { histiocytosis; TB, tuberculosis; ACFE, airway-centered } \\
\text { fibroelastosis; PAM, pulmonary alveolar microlithiasis; PO } \\
\text { pulmonary ossification; IPH, idiopathic pulmonary hemosiderosis. }\end{array}$} \\
\hline
\end{tabular}


Table 4 MDD diagnosis of patients undergoing TBCB

\begin{tabular}{|c|c|}
\hline MDD diagnosis & Case (\%) \\
\hline CTD-ILD & $89(23.9)$ \\
\hline IPF & $33(8.8)$ \\
\hline INSIP & $7(1.9)$ \\
\hline COP & $7(1.9)$ \\
\hline PAP & $15(4.0)$ \\
\hline PAM & $10(2.7)$ \\
\hline Lung cancer & $18(4.8)$ \\
\hline Pneumonia & $11(2.9)$ \\
\hline HP & $6(1.6)$ \\
\hline Sarcoidosis & $5(1.3)$ \\
\hline Pneumoconiosis & $5(1.3)$ \\
\hline Desquamative interstitial pneumonia & $4(1.1)$ \\
\hline TB & $4(1.1)$ \\
\hline Emphysema & $4(1.1)$ \\
\hline RB-ILD & $3(0.8)$ \\
\hline ACFE & $2(0.5)$ \\
\hline PCP & $1(0.3)$ \\
\hline $\mathrm{PLCH}$ & $1(0.3)$ \\
\hline PAM & $1(0.3)$ \\
\hline Cryptococcosis & $1(0.3)$ \\
\hline Occupational-related pneumonia & $1(0.3)$ \\
\hline Radiation pneumonia & $1(0.3)$ \\
\hline Diffuse large B lymphoma in pulmonary vessels & $1(0.3)$ \\
\hline Giant cell interstitial pneumonia & $1(0.3)$ \\
\hline Lipid pneumonia & $1(0.3)$ \\
\hline $\mathrm{IPH}$ & $1(0.3)$ \\
\hline PIE & $1(0.3)$ \\
\hline $\mathrm{PO}$ & $1(0.3)$ \\
\hline DR-ILD & $1(0.3)$ \\
\hline Aspiration pneumonia & $1(0.3)$ \\
\hline ND & $136(36.5)$ \\
\hline
\end{tabular}

MDD, multidisciplinary discussion; CTD, connective tissue disease; ILD, interstitial lung disease; IPF, idiopathic pulmonary fibrosis; iNSIP, idiopathic non-specific interstitial pneumonia; ACFE, airway-centered fibroelastosis; COP, cryptogenic organizing pneumonia; PAP, pulmonary alveolar proteinosis; PLAM, pulmonary lymphangiomyomatosis; HP, hypersensitivity pneumonitis; DIP, desquamative interstitial pneumonia; $\mathrm{PCP}$, pneumocystis pneumonia; PLCH, pulmonary Langerhans cell histiocytosis; RB-ILD, respiratory bronchiolitis-interstitial lung disease; TB, tuberculosis; PAM, pulmonary alveolar microlithiasis; $\mathrm{PO}$, pulmonary ossification; IPH, idiopathic pulmonary hemosiderosis; DR-ILD, drug-related interstitial lung disease.
Table 5 Comparison of technical parameters of TBCB in cases with a definitive diagnosis of MDD and an unclarified diagnosis of MDD

\begin{tabular}{|c|c|c|c|c|}
\hline $\begin{array}{l}\text { Technical } \\
\text { parameters }\end{array}$ & $\begin{array}{l}\text { Clear MDD } \\
\text { diagnosis } \\
\text { (median) }\end{array}$ & $\begin{array}{l}\text { Unknown MDD } \\
\text { diagnosis } \\
\text { (median) }\end{array}$ & $\mathrm{Z} / \chi^{2}$ & $P$ value \\
\hline $\begin{array}{l}\text { Number of } \\
\text { cases (n) }\end{array}$ & 237 & 136 & & \\
\hline $\begin{array}{l}\text { Cooling gas } \\
\text { pressure } \\
\text { (bar) }\end{array}$ & $59.0(55.0,60.0)^{\ddagger}$ & $55.0(54.8,60.0)$ & -4.817 & $\mathrm{P}<0.001$ \\
\hline $\begin{array}{l}\text { Freezing } \\
\text { duration } \\
\text { (seconds) }\end{array}$ & $5.0(4.0,6.0)$ & $5.0(4.0,5.0)$ & -5.595 & $\mathrm{P}<0.001$ \\
\hline $\begin{array}{l}\text { Specimen } \\
\text { length (mm) }\end{array}$ & $4.9(4.0,5.4)$ & $4.2(3.5,5.1)$ & -4.597 & $P<0.001$ \\
\hline $\begin{array}{l}\text { Specimen } \\
\text { short } \\
\text { diameter } \\
(\mathrm{mm})\end{array}$ & $3.6(3.0,4.0)$ & $3.3(3.0,4.0)$ & -4.446 & $P<0.001$ \\
\hline $\begin{array}{l}\text { Specimen } \\
\text { size }\left(\mathrm{mm}^{2}\right)\end{array}$ & $17.6(12.2,21.9)$ & $16.0(9.0,20.2)$ & -4.538 & $P<0.001$ \\
\hline $\begin{array}{l}\text { Number of } \\
\text { specimens }\end{array}$ & $4.0(3.0,5.0)$ & $3.0(3.0,4.0)$ & -4.805 & $P<0.001$ \\
\hline $\begin{array}{l}\text { Navigation, } \\
\text { n (\%) }\end{array}$ & $182(76.8 \%)$ & $64(47.1 \%)$ & 34.023 & $\mathrm{P}<0.001^{\#}$ \\
\hline
\end{tabular}

cryoprobe (2.4-mm cryoprobe group). No serious bleeding occurred in either group of patients. The incidences of pneumothorax in the 1.9 -mm cryoprobe group and the $2.4-\mathrm{mm}$ cryoprobe group were $3.7 \%(12 / 326)$ and $14.9 \%$ (7/47), respectively $(\mathrm{P}<0.05)$. The proportions of patients with a definitive pathological diagnosis in the 1.9-mm cryoprobe group and the 2.4-mm cryoprobe group were $64.4 \%(210 / 326)$ and $48.9 \%(23 / 47)$, respectively. The proportions of patients with a possible pathological diagnosis in the $1.9-\mathrm{mm}$ cryoprobe group and the 2.4-mm cryoprobe group were $5.5 \%(18 / 326)$ and $6.4 \%$ (3/326), respectively. The proportions of patients with an unclarified pathological diagnosis in the $1.9-\mathrm{mm}$ cryoprobe group and the 2.4-mm cryoprobe group were $30.1 \%(98 / 326)$ and $48.9 \%$ (21/47), respectively. There were no significant differences in pathological diagnosis efficiency between the 2 groups $\left(\chi^{2}=4.382 ; \mathrm{P}=0.112\right)$. The proportions of patients who obtained a final definitive 
MDD diagnosis were 65.6\% (214/326) and 48.9\% (23/47), respectively $(\mathrm{P}<0.05$, Table 6$)$.

\section{Comparison of safety and diagnostic efficiency using different specimen quantities}

A total of 1,383 specimens were obtained from 373 patients and the number of specimens obtained from each patient was between 1 and 5 , with an average of $3.7 \pm 1.1$. According to the number of specimens obtained, the patients were divided

Table 6 Comparison of diagnostic efficiencies and ТВСВ complication rates between the $1.9-\mathrm{mm}$ cryoprobe and the $2.4-\mathrm{mm}$ cryoprobe

\begin{tabular}{lcc}
\hline Variable & $1.9-\mathrm{mm}$ & $2.4-\mathrm{mm}$ \\
\hline Number of patients, n (\%) & $326(87.4)$ & $47(12.6)$ \\
Bleeding severity, $\mathrm{n}(\%)$ & & \\
Grade 0 & $53(16.3)$ & $13(27.7)$ \\
Grade 1 & $82(25.2)$ & $8(17.0)$ \\
Grade 2 & $191(58.6)$ & $26(55.3)$ \\
Grade 3 & 0 & 0 \\
Pneumothorax, n (\%) & $12(3.7)$ & $7(14.9)^{\star}$ \\
Pathological diagnosis, n (\%) & & \\
Clear pathological diagnosis & $210(64.4)$ & $23(48.9)$ \\
Possible pathological diagnosis & $18(5.5)$ & $3(6.4)$ \\
Unknown pathological diagnosis & $98(30.1)$ & $21(44.6)$ \\
MDD diagnosis, n (\%) & $214(65.6)$ & $23(48.9)^{\star *}$ \\
\hline
\end{tabular}

*, compared with 1.9-mm group, $\mathrm{P}<0.05$; ${ }^{*}$, compared to the 1.9-mm group, $\mathrm{P}<0.05$. MDD, multidisciplinary discussion. into groups, groups $1-5$. No serious bleeding was observed among the 5 groups of patients. In groups $1-5$, the incidences of pneumothorax were $22.2 \%, 2.3 \%, 6.5 \%, 6.1 \%$, and $2.5 \%$, respectively, and the percentages of definitive MDD diagnosis were $33.3 \%, 43.2 \%, 56.5 \%, 67.3 \%$, and $77.2 \%$, respectively. Significant differences in the diagnostic yield of MDD were found among the 5 groups of patients $\left(\chi^{2}=23.525 ; \mathrm{P}=0.000\right.$, Table 7). The diagnostic yield of MDD was the highest when 5 specimens were obtained, while the complications did not increase significantly.

\section{Discussion}

The recommendation to use TBCB instead of a SLB to diagnose ILD is still controversial (3,4,7-9). TBCB has higher diagnostic efficiency and lower incidence of complications, and it is suitable for the initial biopsy for suspected cases of ILD (10). Previous studies (1,2,10-14) have shown that the diagnostic yield of TBCB for ILD ranges from $72.9 \%$ to $84.4 \%$, the incidence of severe bleeding ranges from $0.3 \%$ to $26.6 \%$, and the incidence of pneumothorax ranges from $6 \%$ to $9.5 \%$. The data show that the diagnostic efficiencies and the complication rates of different studies are extremely variable. Therefore, this prospective multicenter real-world study aimed to explore the actual application of TBCB in ILD in China, with 20 hospitals in 12 provinces or municipalities in China submitting their data. Importantly, this study is the first report on application of TBCB in ILD with a real-world study in China.

We ultimately enrolled 373 patients with an unclarified diagnosis of ILD. Most of the patients had moderate to severe lung diffusion impairment. However, the proportion

Table 7 Comparison of diagnostic efficiencies and complication rates between different numbers of obtained specimens

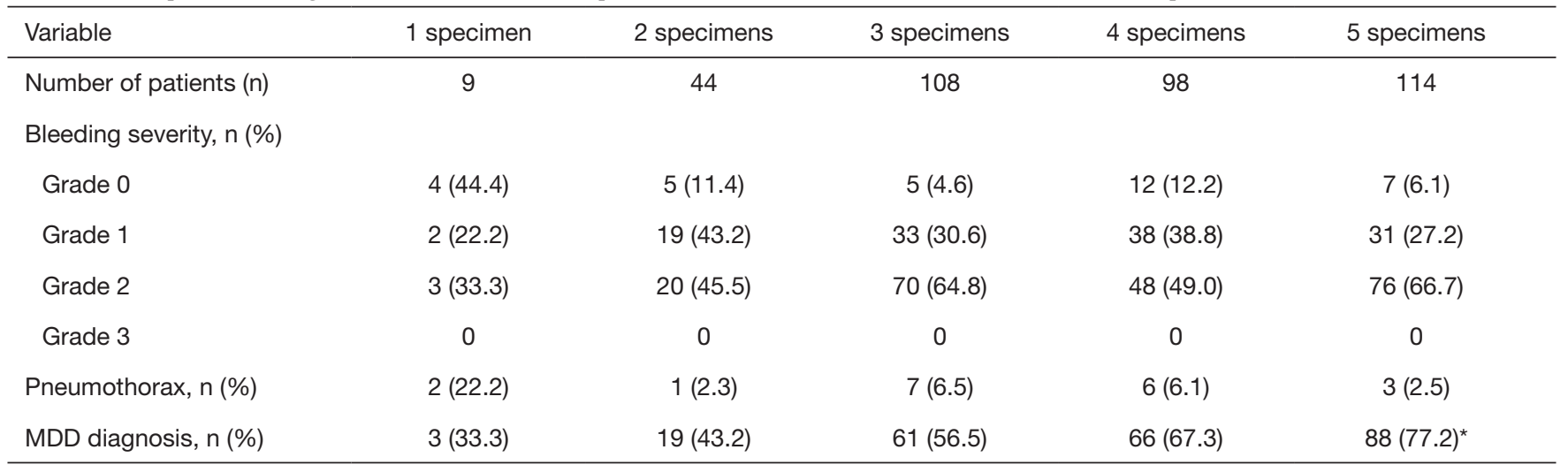

${ }^{*}$, the diagnosis of MDD was significantly different between the 5 groups $\left(\chi^{2}=23.525 ; P<0.001\right)$. MDD, multidisciplinary discussion. 
of moderate bleeding in all patients was reach as high as $57.6 \%$, and these patients needed balloon occlusion or local adrenaline injection to stop bleeding during TBCB. Studies $(15,16)$ have shown that balloon occlusion significantly reduces the incidence of severe bleeding. In this study, some patients also needed balloon occlusion during TBCB, which might have been an important reason why none of the patients had serious fatal bleeding. The incidence of pneumothorax was approximately 5.1\%. Most patients with pneumothorax required closed chest drainage and recovered within a week. The safety of TBCB was consistent with previous reports $(2,17-27)$. There was no relationship between the complication rate of TBCB and the different comorbidities in the patients. The types of disease in the selected cases of this study were also the same as those in previous reports $(1,2,11-14)$ and the most common type of disease was connective tissue disease-ILD and idiopathic pulmonary fibrosis. However, only $63.5 \%$ of the patients obtained a definitive diagnosis of ILD after MDD. These results were significantly lower than the approximately $80 \%$ diagnostic accuracy for ILD in other reports (12-14).

The low diagnostic accuracy of TBCB in real-world in this study may be related to the following aspects. First, the specimen size was smaller than that of other publications $(18,28)$, which is one of the key factors related to a low diagnostic yield. The cooling gas pressure in our study was $54.5 \pm 11.6$ bar and even $<50$ bar in some cases. A previous study (29) has shown the specimen size of TBCB to be positively correlated with the cooling gas pressure. When the cooling gas pressure decreases, its freezing efficiency is also reduced, resulting in a small biopsy specimen and affecting the quality of the specimen if the freezing time is not increased correspondingly. In this study, the freezing time was $4.9 \pm 1.4$ seconds, which was lower than the 7 seconds recommended by the current expert consensus (10). Low cooling gas pressure combined with insufficient freezing time can lead to unsatisfactory tissue specimens, leading to a decrease in the diagnostic yield of TBCB in ILD. Thus, it is necessary to pay attention to the effect of the cooling gas pressure and freezing time on the size and quality of TBCB specimens. Second, we obtained only 1 or 2 specimens in some cases because of pneumothorax or bleeding. The current expert consensus recommends 3 to 5 tissue specimens for pathological diagnosis of ILD (10). A sampling of only 1 to 2 tissue samples may affect the diagnostic yield of ILD. Third, studies have shown that navigation-guided TBCB reduces the incidence of complications and increases the diagnostic yield of TBCB in ILD (2,16,30-33). However, only $66.0 \%$ of the patients in this study underwent navigation-guided TBCB. ILD lesions are mainly distributed around the peripheral lung and subpleura lines. Thus, the inclusion of patients without navigation-guided TBCB might have resulted in the acquisition of nonrepresentative specimens from too central of the lung for diagnosis. Fourth, we divided the patients into the definitive MDD diagnosis group and the indefinite MDD diagnosis group to compare TBCB parameters. Our results showed significant differences in the cooling gas pressure, freezing time, number of specimens, specimen size, and utilization rate of navigation tools between the two groups. The failure to acknowledge these TBCB parameters and their improper use were also important reasons for the low diagnostic efficiency of TBCB in ILD. Lastly, the low diagnostic efficiency of TBCB may be a product of the rapid development of TBCB in China, some operators may not follow a standardized procedure for TBCB. Thus, it is necessary to pay attention to the key parameters of TBCB, including the cooling gas pressure, the freezing time, the number of specimens, and the navigation tools to improve the efficacy and safety of TBCB.

There were $326(87.4 \%)$ patients and 47 (12.6\%) patients in whom TBCB was performed with $1.9-\mathrm{mm}$ and 2.4-mm diameter cryoprobes, respectively. There were no differences in the incidence of bleeding among the patients who underwent TBCB with the 2 different cryoprobes. However, the incidence of pneumothorax in patients in the 2.4-mm cryoprobe group was significantly higher than that in patients in the $1.9-\mathrm{mm}$ cryoprobe group. This result was consistent with the previous report (28) because the 2.4-mm cryoprobe had a higher cooling efficiency in obtaining larger specimens under the same freezing time. The larger the specimens collected were, the higher was the risk of pneumothorax in patients. Although patients who underwent TBCB with a 2.4-mm cryoprobe had a higher complication rate, the diagnostic yield of MDD was lower than that in patients by using a 1.9-mm cryoprobe. It may be that the lesions of ILD were mainly distributed in the subpleural area and the lower lobes and the $1.9-\mathrm{mm}$ cryoprobe was easier to extend to the peripheral lungs for biopsy, thus making it easier to obtain a presentative sample for diagnosis, as compared with a 2.4-mm cryoprobe. Therefore, the $1.9-\mathrm{mm}$ cryoprobe is recommended for TBCB to achieve a better diagnosis and lower the complication rate in ILD.

The number of specimens ideal for the pathological diagnosis of ILD is unknown, because the size and number 
of specimen also affect the final pathological diagnosis of ILD. Current expert consensus recommends obtaining 3 to 5 specimens from 2 different lobes to diagnose ILD in a patient (10). Furthermore, the Indian expert consensus on cryobiopsy also recommends obtaining at least 4 lung specimens to diagnose ILD in a patient (34). This study showed that as the number of specimens increased, the diagnostic yield of TBCB continued to improve. When 5 specimens were acquired, the diagnostic yield was optimized, while the risk of complications did not increase significantly. Although we obtained 3 to 5 specimens for the pathological diagnosis of ILD based on expert consensus, considering the complications and safety of the procedure, we did not take more than 3 specimens for diagnosis in some patients. For example, only 1 specimen was obtained from each of 3 patients with pneumothorax at the first biopsy. However, the collection of only 1 specimen during TBCB was unlikely to cause pneumothorax. Therefore, the correlation between the number of specimens and the complications resulting from TBCB has not been determined. However, the correlation between the number of specimen and the diagnostic yield should be credible. Under the premise of ensuring patient safety, we suggest taking 5 tissue specimens to increase the diagnostic yield of TBCB in ILD.

This was a prospective, real-world study without any interventions applied in the participating units, including restrictions on the cooling gas pressure, anesthesia methods, freezing time, navigation tools, or the number of specimens. This study aimed to clarify the real-world use of TBCB in China. The participating units in this study represent the current application of TBCB in China. However, due to the absence of intervention and control groups, the number of patients in the $1.9-\mathrm{mm}$ cryoprobe group and the 2.4- $\mathrm{mm}$ cryoprobe group, and the number of patients grouped with different specimen numbers in the subgroup, varied greatly. Thus, a multicenter, randomized controlled study should be conducted for further verification in a larger prospective sample size.

\section{Conclusions}

Our real-world study found that the overall safety of TBCB for ILD in China was good, and its diagnostic efficiency was acceptable. To improve the diagnostic yield and safety, establishing standardized operating procedures and standardized training in TBCB is recommended. In addition, TBCB in ILD using a $1.9-\mathrm{mm}$ cryoprobe and collecting 5 specimens may achieve a better positive diagnostic rate without a corresponding increase in complication risk.

\section{Acknowledgments}

Funding: This study was funded by Medical Research Foundation of Guangdong (A2020106).

\section{Footnote}

Reporting Checklist: The authors have completed the STROBE reporting checklist. Available at https://dx.doi. org/10.21037/atm-21-3461

Data Sharing Statement: Available at https://dx.doi. org/10.21037/atm-21-3461

Conflicts of Interest: All authors have completed the ICMJE uniform disclosure form (available at https://dx.doi. org/10.21037/atm-21-3461). The authors have no conflicts of interest to declare.

Ethical Statement: The authors are accountable for all aspects of the work in ensuring that questions related to the accuracy or integrity of any part of the work are appropriately investigated and resolved. The study was conducted in accordance with the Declaration of Helsinki (as revised in 2013). The study was approved by the Ethics Committee of the First Affiliated Hospital of Guangzhou Medical College, Guangdong Province, China (No. 201813) and informed consent was taken from all individual participants.

Open Access Statement: This is an Open Access article distributed in accordance with the Creative Commons Attribution-NonCommercial-NoDerivs 4.0 International License (CC BY-NC-ND 4.0), which permits the noncommercial replication and distribution of the article with the strict proviso that no changes or edits are made and the original work is properly cited (including links to both the formal publication through the relevant DOI and the license). See: https://creativecommons.org/licenses/by-nc-nd/4.0/.

\section{References}

1. Ravaglia C, Bonifazi M, Wells AU, et al. Safety and Diagnostic Yield of Transbronchial Lung Cryobiopsy 
in Diffuse Parenchymal Lung Diseases: A Comparative Study versus Video-Assisted Thoracoscopic Lung Biopsy and a Systematic Review of the Literature. Respiration 2016;91:215-27.

2. Dhooria S, Sehgal IS, Aggarwal AN, et al. Diagnostic Yield and Safety of Cryoprobe Transbronchial Lung Biopsy in Diffuse Parenchymal Lung Diseases: Systematic Review and Meta-Analysis. Respir Care 2016;61:700-12.

3. Romagnoli M, Colby TV, Berthet JP, et al. Poor Concordance between Sequential Transbronchial Lung Cryobiopsy and Surgical Lung Biopsy in the Diagnosis of Diffuse Interstitial Lung Diseases. Am J Respir Crit Care Med 2019;199:1249-56.

4. Raghu G, Remy-Jardin M, Myers JL, et al. Diagnosis of Idiopathic Pulmonary Fibrosis. An Official ATS/ERS/JRS/ ALAT Clinical Practice Guideline. Am J Respir Crit Care Med 2018;198:e44-68.

5. Maldonado F, Danoff SK, Wells AU, et al. Transbronchial Cryobiopsy for the Diagnosis of Interstitial Lung Diseases: CHEST Guideline and Expert Panel Report. Chest 2020;157:1030-42.

6. Ernst A, Eberhardt R, Wahidi M, et al. Effect of routine clopidogrel use on bleeding complications after transbronchial biopsy in humans. Chest 2006;129:734-7.

7. Troy LK, Grainge C, Corte TJ, et al. Diagnostic accuracy of transbronchial lung cryobiopsy for interstitial lung disease diagnosis (COLDICE): a prospective, comparative study. Lancet Respir Med 2020;8:171-81.

8. Tomassetti S, Ravaglia C, Wells AU, et al. Prognostic value of transbronchial lung cryobiopsy for the multidisciplinary diagnosis of idiopathic pulmonary fibrosis: a retrospective validation study. Lancet Respir Med 2020;8:786-94.

9. Hetzel J, Wells AU, Costabel U, et al. Transbronchial cryobiopsy increases diagnostic confidence in interstitial lung disease: a prospective multicentre trial. Eur Respir J

10. Hetzel J, Maldonado F, Ravaglia C, et al. Transbronchial Cryobiopsies for the Diagnosis of Diffuse Parenchymal Lung Diseases: Expert Statement from the Cryobiopsy Working Group on Safety and Utility and a Call for Standardization of the Procedure. Respiration 2018;95:188-200.

11. Johannson KA, Marcoux VS, Ronksley PE, et al. Diagnostic Yield and Complications of Transbronchial Lung Cryobiopsy for Interstitial Lung Disease. A Systematic Review and Metaanalysis. Ann Am Thorac Soc 2016;13:1828-38.

12. Sharp C, McCabe M, Adamali H, et al. Use of transbronchial cryobiopsy in the diagnosis of interstitial lung disease-a systematic review and cost analysis. QJM 2017;110:207-14.

13. Iftikhar IH, Alghothani L, Sardi A, et al. Transbronchial Lung Cryobiopsy and Video-assisted Thoracoscopic Lung Biopsy in the Diagnosis of Diffuse Parenchymal Lung Disease. A Meta-analysis of Diagnostic Test Accuracy. Ann Am Thorac Soc 2017;14:1197-211.

14. Sethi J, Ali MS, Mohananey D, et al. Are Transbronchial Cryobiopsies Ready for Prime Time?: A Systematic

Review and Meta-Analysis. J Bronchology Interv Pulmonol 2019;26:22-32.

15. Echevarria-Uraga JJ, Pérez-Izquierdo J, García-Garai $\mathrm{N}$, et al. Usefulness of an angioplasty balloon as selective bronchial blockade device after transbronchial cryobiopsy. Respirology 2016;21:1094-9.

16. Dhooria S, Mehta RM, Srinivasan A, et al. The safety and efficacy of different methods for obtaining transbronchial lung cryobiopsy in diffuse lung diseases. Clin Respir J 2018;12:1711-20.

17. Gershman E, Fruchter O, Benjamin F, et al. Safety of Cryo-Transbronchial Biopsy in Diffuse Lung Diseases: Analysis of Three Hundred Cases. Respiration 2015;90:40-6.

18. Casoni GL, Tomassetti S, Cavazza A, et al. Transbronchial lung cryobiopsy in the diagnosis of fibrotic interstitial lung diseases. PLoS One 2014;9:e86716.

19. Pajares V, Torrego A, Puzo C, et al. Transbronchial lung biopsy using cryoprobes. Arch Bronconeumol 2010;46:111-5.

20. Griff S, Ammenwerth W, Schönfeld N, et al. Morphometrical analysis of transbronchial cryobiopsies. Diagn Pathol 2011;6:53.

21. Kropski JA, Pritchett JM, Mason WR, et al. Bronchoscopic cryobiopsy for the diagnosis of diffuse parenchymal lung disease. PLoS One 2013;8:e78674.

22. Fruchter O, Fridel L, Rosengarten D, et al. Transbronchial cryobiopsy in immunocompromised patients with pulmonary infiltrates: a pilot study. Lung 2013;191:619-24.

23. Fruchter O, Fridel L, Rosengarten D, et al. Transbronchial cryo-biopsy in lung transplantation patients: first report. Respirology 2013;18:669-73.

24. Fruchter O, Fridel L, El Raouf BA, et al. Histological diagnosis of interstitial lung diseases by cryotransbronchial biopsy. Respirology 2014;19:683-8.

25. Griff S, Schönfeld N, Ammenwerth W, et al. Diagnostic yield of transbronchial cryobiopsy in non-neoplastic lung disease: a retrospective case series. BMC Pulm Med 2014;14:171. 
26. Hagmeyer L, Theegarten D, Wohlschläger J, et al. The role of transbronchial cryobiopsy and surgical lung biopsy in the diagnostic algorithm of interstitial lung disease. Clin Respir J 2016;10:589-95.

27. Hernández-González F, Lucena CM, Ramírez J, et al. Cryobiopsy in the diagnosis of diffuse interstitial lung disease: yield and cost-effectiveness analysis. Arch Bronconeumol 2015;51:261-7.

28. Ravaglia C, Wells AU, Tomassetti S, et al. Diagnostic yield and risk/benefit analysis of trans-bronchial lung cryobiopsy in diffuse parenchymal lung diseases: a large cohort of 699 patients. BMC Pulm Med 2019;19:16.

29. Chen X, Li S, Xiao W, et al. Applied pressure of cooling gas: a neglected determinant for size of specimens in transbronchial cryobiopsy. J Thorac Dis 2021;13:2099-105.

30. Gnass M, Filarecka A, Bartczak A, et al. Transbronchial lung cryobiopsy guided by radial mini-probe endobronchial

Cite this article as: Chen $\mathrm{X}$, Li J, Luo F, Tao F, Zhang X, Wu Y, Xu P, Ke M, Long F, Liu L, Lv L, Liao H, Gu Y, Liu Z, Tan X, Guo S, Hu Y, Yang H, Zhou Y, Zhou H, Ye Y, Chen D, Li S. The application of transbronchial cryobiopsy in interstitial lung disease: a prospective, multicenter, real-world study. Ann Transl Med 2021;9(22):1645. doi: 10.21037/atm-21-3461 ultrasound in interstitial lung diseases - a multicenter prospective study. Adv Respir Med 2020;88:123-8.

31. Taton O, Bondue B, Gevenois PA, et al. Diagnostic Yield of Combined Pulmonary Cryobiopsies and Electromagnetic Navigation in Small Pulmonary Nodules. Pulm Med 2018;2018:6032974.

32. Wijmans L, Bonta PI, Rocha-Pinto R, et al. Confocal Laser Endomicroscopy as a Guidance Tool for Transbronchial Lung Cryobiopsies in Interstitial Lung Disorder. Respiration 2019;97:259-63.

33. Chang CH, Lee CS, Li SH, et al. Feasibility of Radial Endobronchial Ultrasound-Guided Bronchoscopic Cryobiopsy without Fluoroscopy for Lung Parenchymal Lesions. Can Respir J 2017;2017:7170687.

34. Dhooria S, Agarwal R, Sehgal IS, et al. Bronchoscopic lung cryobiopsy: An Indian association for bronchology position statement. Lung India 2019;36:48-59. 